\title{
Novel Solution- and Solid-Phase Syntheses of Heterocyclic Systems
}

\author{
Gaelle Cabon, Berangere Gaucher, Aline Gegout, Sophie Heulle, and Thierry Masquelin*
}

\begin{abstract}
Heterocyclic compounds are an attractive source of screening library structures because they possess varied structural diversity and can exhibit potent biological activity. In this context, we present some of our new and versatile approaches to rapid and efficient syntheses of pharmacologically relevant core structures. These include: combination of both solution- and solid-phase processes in the synthesis of pyrazolo[1,5-a]-[1,3,5]-triazin-4-ones and pyrazolo[1,5-a]-[1,3,5]-triazines; parallel, multi-generation synthesis of highly functionalized heterocyclic compounds in solution; a multi-step synthesis of 2,5-diketopiperazine on solid support taking advantage of a bicyclic $\beta$-lactam scaffold, and a combined solid- and solution-phase synthesis of a new class of 2,4-diaminothiazoles.
\end{abstract}

Keywords: Bicyclic $\beta$-lactam · Combined solution- and solid-phase processes · Heterocycles · Parallel multi-generation synthesis

\section{Introduction}

The dramatic developments in molecular biology and high-throughput screening over the past 30 years have intensified the demand for highly diverse libraries of low-molecular weight organic molecules. One way to meet this demand is through appropriate application of combinatorial chemistry. The term combinatorial chemistry implies: generation of a chemical library, a deconvolution strategy, and a linked high-throughput screening system. Four general approaches have been developed for the purpose of synthesis and evaluation of chemical libraries: (a) libraries derived from biological sources [1-3]; (b) a parallel synthetic approach using solutionphase or solid-phase methodologies or a combination of the two; (c) 'split and mix'

\footnotetext{
${ }^{*}$ Correspondence: Dr. T. Masquelin Combinatorial \& Parallel Chemistry Basilea Pharmaceutica Ltd PO Box 3255

synthesis; (d) affinity binding selection approach [1-3]. The original idea of combinatorial chemistry randomly synthesizing a plethora of compounds, often as mixtures, with the associated belief that solely high numbers could speed up the drug development process was a dream-like concept for many chemists. It took several years before scientists realized that it was not only the number of compounds but also their quality and diversity that was essential for proper progress in medicinal chemistry. The prevailing approach today is based on both solution- and solid-phase chemistry directed towards the synthesis of discrete, highpurity compounds. Combinatorial chemistry became a standard tool in medicinal chemistry. Nowadays, medicinal chemists recognize its power in delivering the targeted compounds in a much faster way, and in acceptable quantities and purities.

Initial-phase combinatorial chemistry is applied to discover lead compounds rapidly, which are then subjected to lead validation, followed by lead optimization to produce drug candidates. Since heterocyclic products are pharmacologically rich, possess varied structural diversity, and exhibit potent biological activity, they are an attractive source of library structures. In this context, one major focus of our activities is the development of new solution- and solid-phase access to a diverse array of lowmolecular weight heterocyclic compounds.
We present below some of our new approaches to rapid and efficient syntheses of pharmacologically relevant core structures; combination of solution- and solidphase processes in the synthesis of pyrazolo[1,5-a]-[1,3,5]-triazin-4-ones and pyrazolo[1,5-a]-[1,3,5]-triazines; parallel multigeneration synthesis of highly functionalized heterocycles in solution; a multi-step synthesis of 2,5-diketopiperazine on a solid support taking advantage of a bicyclic $\beta$-lactam scaffold, and finally a combined solid-and solution-phase synthesis of a new class of 2,4-diaminothiazoles.

\section{Pyrazolo[1,5-a]-[1,3,5]-triazin- 4-ones and Pyrazolo[1,5-a]-[1,3,5]- triazines}

Pyrazoles and fused pyrazolo-heterocycles are common components of a large number of natural products and pharmacologically active molecules. Despite intense synthetic interest in these heterocyclic classes, and a number of unique approaches to satisfy the ever-growing need for new chemical entities with inherent pharmacological activity, there is still a need for new general procedures. Thus, in an extension of our studies towards the synthesis of versatile heterocycles on solid-supports, we have established a new general access to pyrazolo[1,5-a]-[1,3,5]-triazin-4-ones and 
pyrazolo[1,5- $a]-[1,3,5]$-triazines of types 8 and 11 (Scheme 1). The production of our libraries starts with key polymerbound $3 \mathrm{H}$-pyrazolo[1,5- $a]$-[1,3,5]-triazin4-one intermediates $\mathbf{4}$ synthesized as outlined in Scheme 1. Thus, condensation of pyrazoles $\mathbf{1}$ with ethoxycarbonyl isothiocyanate 2 in dry acetone at $65^{\circ} \mathrm{C}$, followed by treatment with sodium ethanolate $(1 \mathrm{~N} \mathrm{EtONa} / \mathrm{EtOH})$ in $\mathrm{EtOH}$ at $65{ }^{\circ} \mathrm{C}$ gave $3 \mathrm{H}$-pyrazolo[1,5-a]-[1,3,5]-triazin-4-ones [4], easily attached onto support, using commercially available Merrifield resin $3(1.8 \mathrm{mmol} / \mathrm{g})$ [5-7], in the presence of $\mathrm{N}$-ethyldiisopropylamine (DIPEA; 3 equiv.) in dry $\mathrm{N}, \mathrm{N}$-dimethylformamide (DMF) at $65{ }^{\circ} \mathrm{C}$ (the formation of the resin-bound compounds of type $\mathbf{4}$ was followed by ATR/FT-IR [8]). In this context, the solidphase attachment is used to both purify the diverse 3H-pyrazolo[1,5-a]-[1,3,5]-triazin4-ones using a scavenging process, and introduce new points of diversity taking advantages of the sulfur-based safety catch linkage.
At this stage, we first investigated the reaction of the polymer-bound 3H-pyrazolo[1,5- $a$ ]-[1,3,5]-triazin-4-ones 4 with alkylhalides 5 in DMF at $85{ }^{\circ} \mathrm{C}$, in the presence of 1-tert-butyl-4,4,4-tris-(dimethylamino)2,2-bis-[tris-(dimethylamino)phosphoranenylideamino $]-2 \lambda^{5}, 4 \lambda^{5}$-catenadi-(phosphazene) (phosphazene; 3.2 equiv.), followed by oxidation with 1.3 equiv. of $\mathrm{N}$-(phenylsulfonyl)-3-phenyloxaziridine [9] and subsequent cleavage with various amines 7 in dioxane at $65{ }^{\circ} \mathrm{C}$, leading to 2-amino-3substituted-pyrazolo[1,5- $a]$-[1,3,5]-triazin4-ones of type $\mathbf{8}$ in good yields and high purities (Table 1).

Additionally, treatment of the polymerbound 3H-pyrazolo[1,5- $a]$-[1,3,5]-triazin4-ones 4 with phosphorous oxychloride $\left(\mathrm{POCl}_{3}\right)$ in toluene in the presence of DIPEA at $110{ }^{\circ} \mathrm{C}$, afforded the corresponding 4-chloro intermediates, which underwent nucleophilic substitution with amines 9 to furnish 4-amino polymerbound pyrazolo[1,5-a]-[1,3,5]-triazines $\mathbf{1 0}$.
Finally, oxidation of $\mathbf{1 0}$ with 1.3 equiv of $\mathrm{N}$-(phenylsulfonyl)-3-phenyloxaziridine, followed by cleavage from the support with amines 7, allow us to generate 2,4-diaminopyrazolo[1,5-a]-[1,3,5]-triazines of type $\mathbf{1 1}$ in good yields and high purities (Table 2). The developed strategy appeared ideally suited for the parallel synthesis of a diverse array of low-molecular weight heterocyclic compounds on solid-phase.

\section{Parallel, Multi-generation Synthesis of Highly Functionalized Heterocycles in Solution}

Among the several possible approaches to carry out successfully combinatorial organic synthesis, we also focused our attention on multi-component and multi-generation solution-phase approaches towards five-membered heterocycles and their conversion into fused heterocycles. The success of every combinatorial chemistry approach

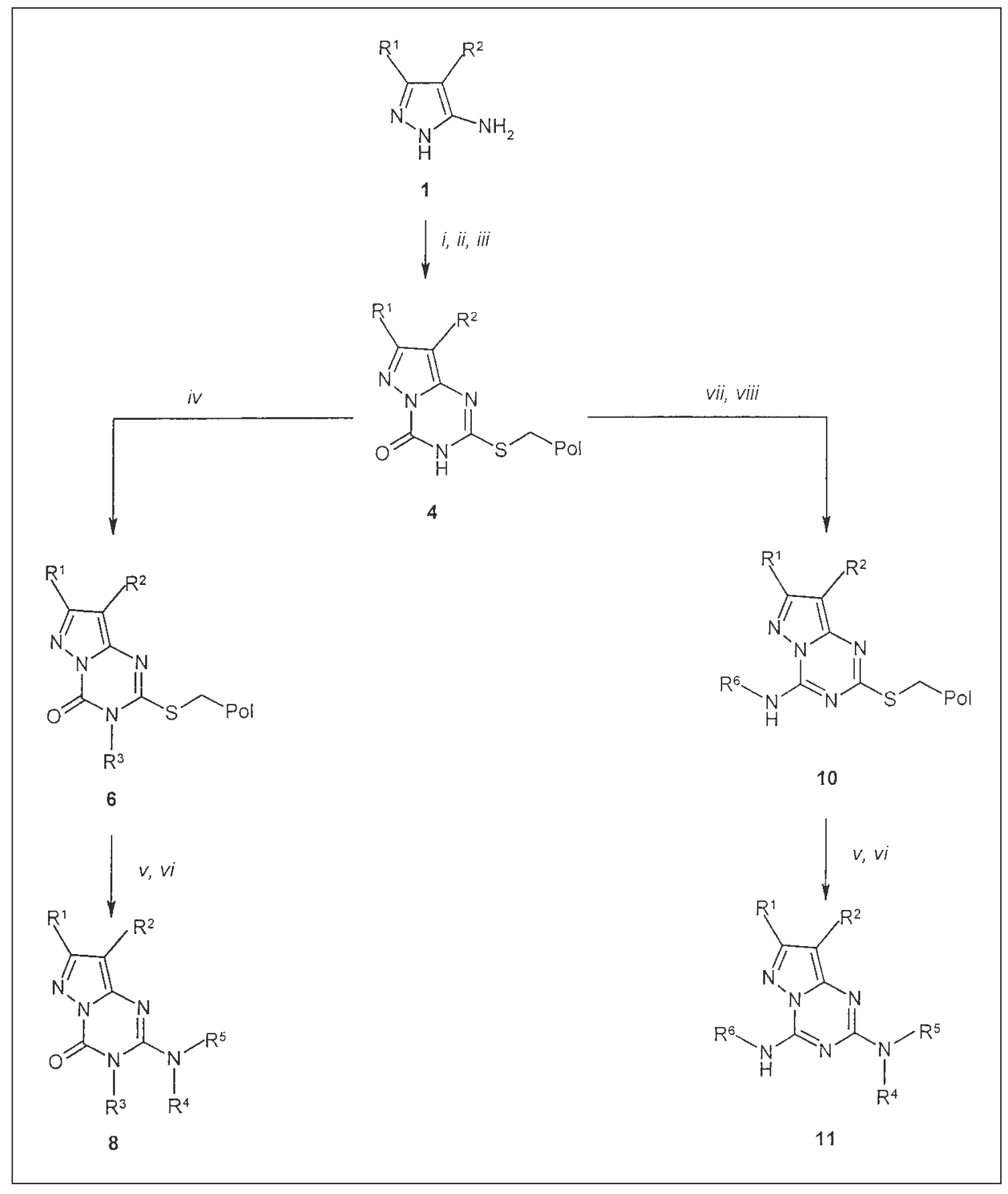

Scheme 1. Reagents and conditions: i) $\mathrm{EtO}_{2} \mathrm{C}$ NCS (2), acetone, rt-65 ${ }^{\circ} \mathrm{C}$; ii) EtONa, EtOH, rt-65 ${ }^{\circ} \mathrm{C}$; iii) Merrifield resin (3), DIPEA, DMF, rt-65 ${ }^{\circ} \mathrm{C}$; iv) $\mathrm{R}^{3}-\mathrm{X}(5)$, phosphazene, DMF, rt$85^{\circ} \mathrm{C}$; v) $\mathrm{CHCl}_{3}$, oxaziridine, rt; vi) $\mathrm{R}^{4} \mathrm{R}^{5}-\mathrm{NH}(7)$, diox., rt-65 ${ }^{\circ} \mathrm{C}$; vii) $\mathrm{POCl}_{3}$, DIPEA, tol., $110^{\circ} \mathrm{C}$; viii) $\mathrm{R}^{6}-\mathrm{NH}_{2}(9)$, diox., $\mathrm{rt}-65^{\circ} \mathrm{C}$ 
Table 1. Preparation of pyrazolo[1,5a]-[1,3,5]-triazin-4-ones 8.

Products $\begin{gathered}\left.\begin{array}{c}\text { Yield }^{a} / \mathrm{Purity}^{b} \\ {[\%]}\end{array}\right] \\ \mathrm{H}\end{gathered}$

aYields in \% are based on weight of crude material and are relative to the initial loading. ${ }^{b} \mathrm{HPLC}$ Purity of the purified material (confirmed by ${ }^{1} \mathrm{H}-\mathrm{NMR}$ ), measured on YMC-Pack Pro $\mathrm{C} 18$ column $(75 \times 4.6 \mathrm{~mm})$ with a gradient $12 \% \mathrm{AcCN} / \mathrm{H}_{2} \mathrm{O} \rightarrow 95 \% \mathrm{AcCN}$ within $5.4 \mathrm{~min}$; flow rate, $2.64 \mathrm{ml} / \mathrm{min}$; UV detection at $200-300 \mathrm{~nm}$.

Table 2. Preparation of pyrazolo[1,5a]-[1,3,5]-triazines 11 .

\begin{tabular}{|c|c|c|c|c|c|}
\hline $\mathrm{R}^{1}$ & $\mathrm{R}^{2}$ & $\mathrm{R}^{4} \mathrm{R}^{5} \mathrm{~N}-$ & $\mathrm{R}^{6} \mathrm{NH}-$ & Products & $\begin{array}{c}\text { Yield }^{a} / \text { Purity }^{b} \\
{[\%]}\end{array}$ \\
\hline & $\mathrm{H}$ & & $\mathrm{NH}_{2}$ & $11 \mathrm{a}$ & $75 / 96$ \\
\hline & $\mathrm{H}$ & & $\mathrm{NH}_{2}$ & $11 b$ & $80 / 99.5$ \\
\hline Me-S & $\mathrm{CN}$ & $\mathrm{NH}_{2}$ & & $11 \mathrm{c}$ & $77 / 95$ \\
\hline Me-S & $\mathrm{CN}$ & $\mathrm{NH}_{2}$ & & 11d & $83 / 99$ \\
\hline $\mathrm{Me}$ & $\mathrm{H}$ & & & $11 \mathrm{e}$ & $67 / 92$ \\
\hline $\mathrm{Me}$ & $\mathrm{H}$ & & & $11 f$ & $67 / 94$ \\
\hline
\end{tabular}

aYields in $\%$ are based on weight of crude material and are relative to the initial loading. ${ }^{b} \mathrm{HPLC}$ Purity of the purified material (confirmed by ${ }^{1} \mathrm{H}-\mathrm{NMR}$ ), measured on YMC-Pack Pro $\mathrm{C} 18$ column $(75 \times 4.6 \mathrm{~mm})$ with a gradient $12 \% \mathrm{AcCN} / \mathrm{H}_{2} \mathrm{O} \rightarrow 95 \% \mathrm{AcCN}$ within $5.4 \mathrm{~min}$; flow rate, $2.64 \mathrm{ml} / \mathrm{min}$; UV detection at $200-300 \mathrm{~nm}$. is critically dependent on the availability of the necessary building blocks. As shown in Scheme 2, we selected ethyl-2-amino cyano acetate 13, a multifunctional central building block, easily accessible by the reduction of ethyl cyano-(hydroxyimino) acetate 12 [10-12], to generate a palette of pharmacologically relevant core structures, such as thiazoles 15, oxazoles 20 and imidazolones 24, with additional elements of structural diversity.

Thus, we describe in Scheme 2 a rapid entry into a series of polyfunctionalized thiazoles 15 , easily obtained in pure form by simple precipitation, in good yields and purities (Table 3) starting from ethyl-2amino cyano acetate $\mathbf{1 3}$ and isothiocyanates 14. Subsequent condensation with ethoxycarbonyl-isothiocyanate $\mathbf{2}$ and treatment with sodium ethanolate in $\mathrm{EtOH}$ at $75^{\circ} \mathrm{C}$, led to the corresponding fused heterocycles of type $\mathbf{1 6}$ in pure form after acidification, precipitation and filtration of the suspension (Table 3). S-alkylation of $\mathbf{1 6}$ with MeI performed in acetone with $\mathrm{Cs}_{2} \mathrm{CO}_{3}$, followed by chlorination with $\mathrm{POCl}_{3}$ in toluene in the presence of DIPEA at $110^{\circ} \mathrm{C}$, afforded the corresponding 4-chloro intermediates, which underwent nucleophilic substitution with amines $\mathbf{1 7}$ to furnish compounds of type $\mathbf{1 8}$ in pure form (Table 4). At this stage removal of solution-phase excess reactants, reagents, and byproducts is accomplished by incubation with CMR/R resins (Complementary Molecular Reactivity and Recognition resins: [13]) and filtration of the reaction mixture. The $\mathrm{CMR} / \mathrm{R}$ library purification strategy is general and highly amenable to automation.

In order to explore in depth the potential of our building block $\mathbf{1 3}$ to generate highly functionalized heterocycles libraries, we studied the reaction of $\mathbf{1 3}$ with acyl chlorides of type 19 under standard conditions and subjected the corresponding amide to acid-catalyzed cyclization (Scheme 2), affording amino-oxazoles 20 in good overall yields and purities (Table 5). At this stage, applying the previously described process: condensation with ethoxycarbonylisothiocyanate $\mathbf{2}$, and treatment with sodium ethanolate led to the corresponding fused heterocycles of type $\mathbf{2 1}$ (Table 5). Finally S-alkylation of 21, followed by chlorination and nucleophilic substitution yielded compounds of type $\mathbf{2 2}$ in pure form, using the $\mathrm{CMR} / \mathrm{R}$ library purification strategy (Table 4).

Additionally to demonstrate the versatility of our building block 13 , we condensed $\mathbf{1 3}$ with isocyanates $\mathbf{2 3}$, and submitted the corresponding intermediates to an acid-catalyzed cyclization, allowing us to generate imidazolones of type 24. Fused 


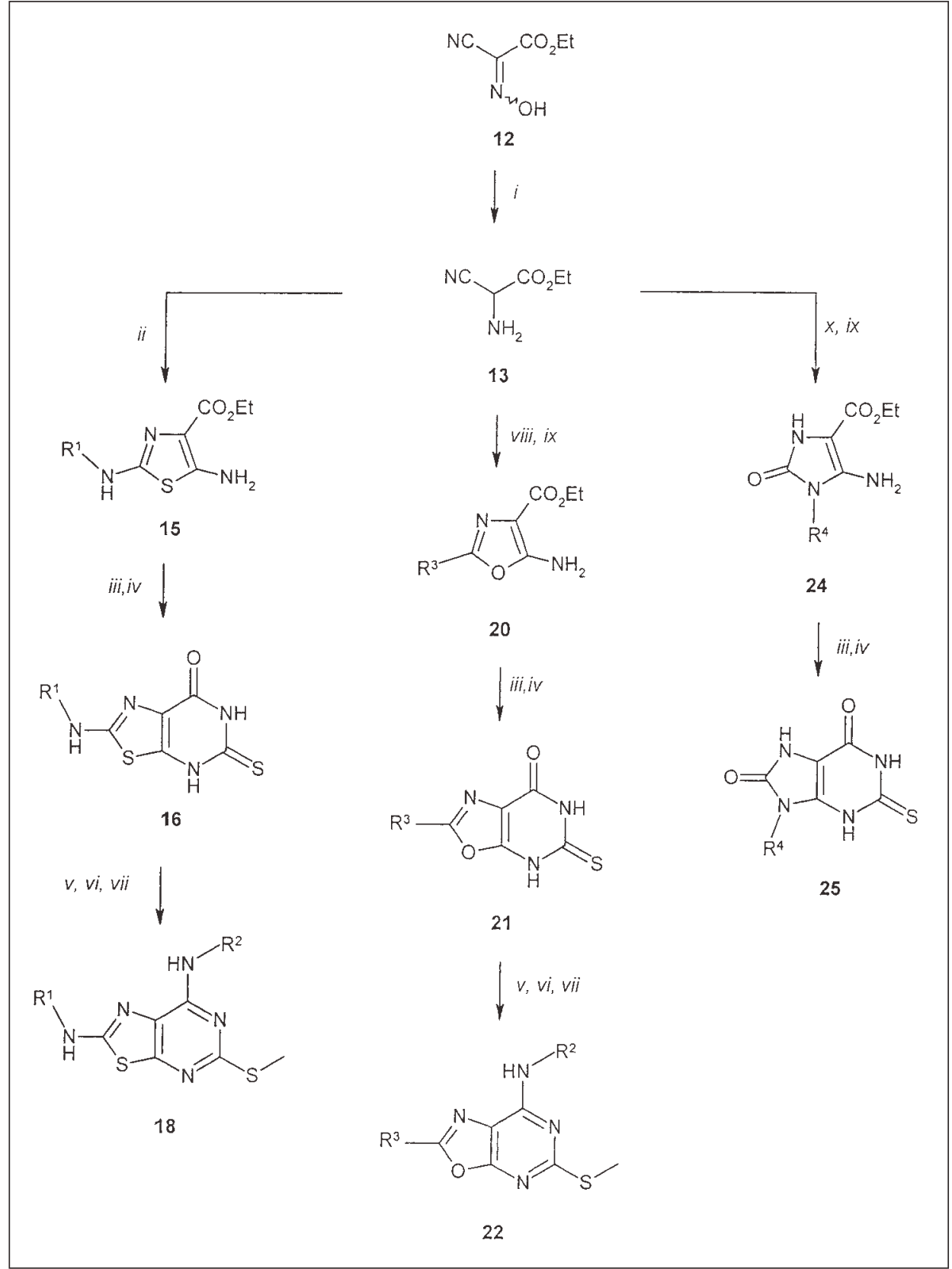

heterocycles $\mathbf{2 5}$ were generated applying the previously described modifications to 24 (Table 6). The described parallel solution multigeneration strategies combined with the $\mathrm{CMR} / \mathrm{R}$ library purification method could be fully automated and performed on a robotic system.

\section{A Multistep Synthesis of 2,5-Diketopiperazine on Solid- Support Taking Advantage of a Bicyclic $\beta$-Lactam Scaffold}

Among the broad range of heterocyclic scaffolds representing lead structures for discovery of potential pharmacophores, we next focused our attention on a new solidphase synthesis of diketopiperazine deriv-
Scheme 2. Reagents and conditions: $i) \mathrm{Na}_{2} \mathrm{~S}_{2} \mathrm{O}_{4}$ $\mathrm{H}_{2} \mathrm{O}$, rt; ii) $\mathrm{R}^{1}$-NCS (14) diox.; iii) $\mathrm{EtO}_{2} \mathrm{C}-\mathrm{NCS}$ (2), acetone, rt-85 ${ }^{\circ} \mathrm{C}$; iv) EtONa, EtOH, $\mathrm{rt}^{-}-75^{\circ} \mathrm{C}$; v) Mel, $\mathrm{Cs}_{2} \mathrm{CO}_{3}$, acetone, rt; vi) $\mathrm{POCl}_{3}$, DIPEA, tol., $110{ }^{\circ} \mathrm{C}$; vii) $\mathrm{R}^{2}-\mathrm{NH}_{2}$ (17), diox., rt-95 ${ }^{\circ} \mathrm{C}$; viii) $\mathrm{R}^{3}-\mathrm{COCl}$ (19), TEA, $\mathrm{CH}_{2} \mathrm{Cl}_{2}$, rt; ix) $\mathrm{HCl} \mathrm{g}$, diox., rt; $x$ ) $\mathrm{R}^{4}-\mathrm{NCO}$ (23), diox., rt-65 ${ }^{\circ} \mathrm{C}$

with amino acids $\mathbf{3 0}$ in DMF to yield the polymer-bound di-substituted pyrrolidines 31. At this stage, deprotection of the pyrrolidine nitrogen carried out using tetrakis(triphenylphosphine) palladium $\left(\mathrm{Pd}\left(\mathrm{PPh}_{3}\right)_{4}\right)$ together with a borane dimethylamine complex $\left(\mathrm{Me}_{2} \mathrm{NH}^{*} \mathrm{BH}_{3}\right)$ [17] furnished the free amine intermediate, which underwent a base-catalyzed cyclization using tetramethyl guanidine (TMG) in DMF at $60{ }^{\circ} \mathrm{C}$ to afford the corresponding polymer-bound diketopiperazines of type 32. The compounds could be easily cleaved off the resin under standard conditions to yield the final products $\mathbf{3 3}$ in good yields and high purities (Table 7).

Additionally a third vector of diversity was introduced onto $\mathbf{3 2}$, by alkylation with alkyl halides 34 in the presence of phosphazene. The resulting diketopiperazines of type $\mathbf{3 5}$ are easily cleaved off the resin. This strategy allows for a rapid synthesis of libraries of diketopiperazines on solidsupport and demonstrates the potential of the bicyclic $\beta$-lactam building block, which can also be used in many different ways for the construction of pyrrolidine libraries. products, diketopiperazine contributes interesting therapeutic properties [14]. Our solid-phase strategy is based on the use of the bicyclic $\beta$-lactam 27 as starting material, which allows the introduction of a large variety of building blocks, and contains different potential attachment points for solidphase. In our approach the scaffold 27 was attached via the alcohol function to a Wang resin activated as trichloroacetamidate $\mathbf{2 6}$ [15][16] to finally obtain the resin-bound bicyclic $\beta$-lactam 28 (Scheme 3 ). Therefore, the $\beta$-lactam nitrogen was acylated with acid-chlorides 29 in DMF in the presence of DIPEA (5 equiv.) and dimethylaminopyridine (DMAP; 2 equiv.) allowing the introduction of the first diversity vector, followed by the opening of the intermediates

\section{Combined Solid- and Solution- phase Synthesis of a New Class of 2,4-Diaminothiazoles and Fused Heterocycles}

Solid-phase synthesis of small organic molecules has emerged as an important tool in drug discovery. Another example of a successful application of solid-phase chemistry constitutes the highly versatile synthesis of 2,4-diaminothiazoles, which is an important heterocyclic nucleus in medicinal chemistry. Our multigeneration strategy combines the cyclocondensation reaction 
Table 3. Preparation of 2,5-diaminothiazole 15 and fused heterocycles 16.

Yield $^{a} /$ Purity $^{b}$
$[\%]$

Table 4. Preparation of fused heterocycles 18 and 22

Yield Purity $^{b}$
$[\%]$

Table 5. Preparation of 5-amino-oxazoles $\mathbf{2 0}$ and fused heterocycles $\mathbf{2 1}$.

\begin{tabular}{|c|c|c|c|c|}
\hline \multirow[t]{6}{*}{$\mathrm{R}^{3}-\mathrm{COCl}$} & Products & Yield/Purity [\%] & Products & $\begin{array}{c}\text { Yield }^{a} / \text { Purity }^{b} \\
{[\%]}\end{array}$ \\
\hline & $20 \mathrm{a}$ & $76 / 99$ & $21 a$ & $22 / 99$ \\
\hline & $20 \mathrm{~b}$ & $51 / 98$ & $21 b$ & $38 / 99$ \\
\hline & $20 \mathrm{c}$ & $71 / 80$ & $21 c$ & $35 / 99$ \\
\hline & $20 \mathrm{~d}$ & $74 / 93$ & 21d & $42 / 99$ \\
\hline & $20 \mathrm{e}$ & $36 / 96$ & $21 e$ & $27 / 99$ \\
\hline
\end{tabular}

of a polymer-bound thiouronium salt $\mathbf{3 6}$ with isothiocyanates $37, \alpha$-bromoketones 38 using a polymer-supported auto-scavenging (PSAS) purification strategy and a solution-phase intramolecular cyclization. Thus, when resin-bound thiouronium salt 36, easily prepared by reaction of thiourea with commercially available Merrifield resin, was allowed to react with isothiocyanates 37 in DMF in the presence of DIPEA as well as N,N-diethylaminomethyl polystyrene resin, and followed by condensation with $\alpha$-bromoketones $\mathbf{3 8}$, the corresponding 2,4-diaminothiazoles 39 were formed in high yields and purities (Scheme 4; Table 8). At this stage to be able to perform the desired intramolecular annelation, deprotection of the Boc group was carried out efficiently by the use of Amberlyst A-15 resin. With the free amines $\mathbf{4 0}$ in hand, the intramolecular cyclization was performed with carbonyl diimidazole (CDI) in DMF in the presence of triethyl amine $\left(\mathrm{Et}_{3} \mathrm{~N}\right)$ to generate the final products $\mathbf{4 1}$ in moderate yields and high purities (Table 8 ). The synthesis of a small library of fused heterocycles as outlined in Scheme 4 was carried out successfully.

\section{Conclusion}

In conclusion, we have demonstrated the capability of combinatorial chemistry to produce rapidly and efficiently a diverse array of low-molecular weight heterocycle compounds using solution- and solid-phase strategies. The payoff of combinatorial chemistry to drug discovery is already becoming obvious in terms of significant increase in the number of lead candidates and time savings from lead candidate identification to validation.

For all Tables:

aYields in $\%$ are based on weight of crude material and are relative to the initial loading. ${ }^{b}$ HPLC Purity of the purified material (confirmed by ${ }^{1} \mathrm{H}$-NMR), measured on YMC-Pack Pro $\mathrm{C} 18$ column $(75 \times 4.6 \mathrm{~mm})$ with a gradient $12 \% \mathrm{AcCN} / \mathrm{H}_{2} \mathrm{O} \rightarrow 95 \% \mathrm{AcCN}$ within $5.4 \mathrm{~min}$; flow rate, $2.64 \mathrm{ml} / \mathrm{min}$; UV detection at 200$300 \mathrm{~nm}$. 
Table 6. Preparation of imidazolones $\mathbf{2 4}$ and fused heterocycles 25.

\begin{tabular}{llccc}
\hline $\mathrm{R}^{4}-\mathrm{NCO}$ & Products & Yield/Purity [\%] & Products & $\begin{array}{c}\text { Yield }^{a} / \text { Purity }^{b} \\
{[\%]}\end{array}$ \\
\hline & $\mathbf{2 4 a}$ & $97 / 99$ & $\mathbf{2 5 a}$ & $60 / 99$ \\
& $\mathbf{2 4 b}$ & $21 / 92$ & $\mathbf{2 5 b}$ & $48 / 99$ \\
\hline
\end{tabular}

aYields in $\%$ are based on weight of crude material and are relative to the initial loading. ${ }^{b}$ HPLC Purity of the purified material (confirmed by $\left.{ }^{1} \mathrm{H}-\mathrm{NMR}\right)$, measured on YMC-Pack Pro C18 column $(75 \times 4.6 \mathrm{~mm})$ with a gradient $12 \% \mathrm{AcCN} / \mathrm{H}_{2} \mathrm{O} \rightarrow 95 \% \mathrm{AcCN}$ within $5.4 \mathrm{~min}$; flow rate, $2.64 \mathrm{ml} / \mathrm{min}$; UV detection at 200-300 nm
Table 7. Preparation of diketopiperazines $\mathbf{3 3}$ and 35.

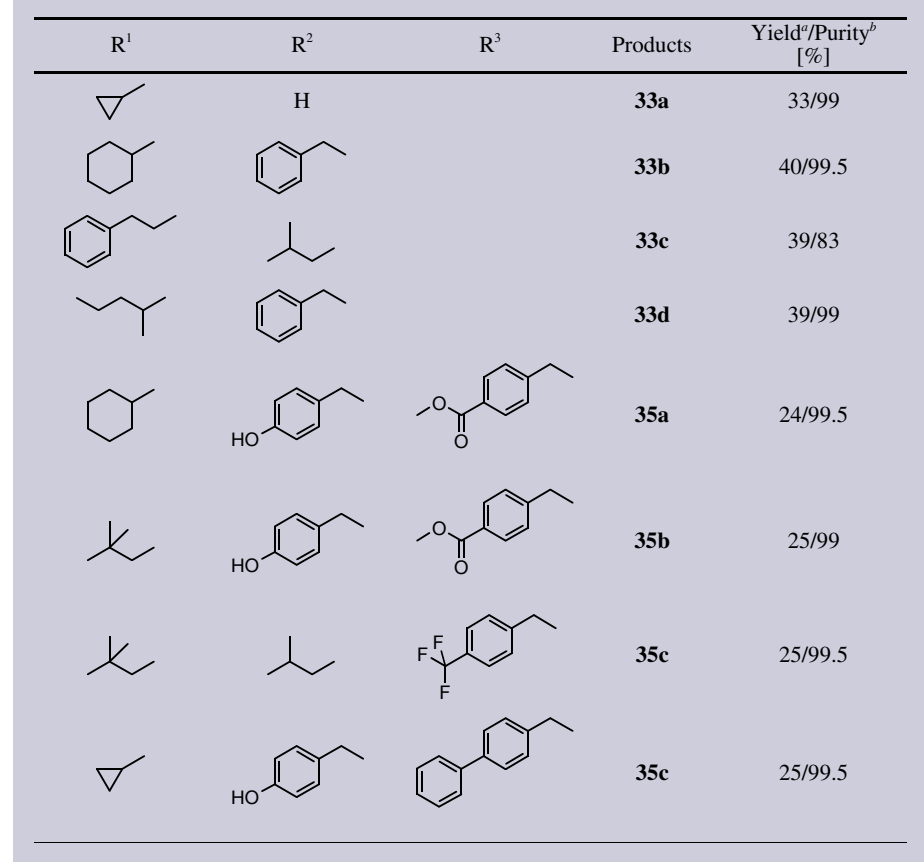

aYields in \% are based on weight of crude material and are relative to the initial loading. ${ }^{b} \mathrm{HPLC}$ Purity of the purified material (confirmed by $\left.{ }^{1} \mathrm{H}-\mathrm{NMR}\right)$, measured on YMC-Pack Pro C18 column $(75 \times 4.6 \mathrm{~mm})$ with a gradient $12 \% \mathrm{AcCN} / \mathrm{H}_{2} \mathrm{O} \rightarrow 95 \% \mathrm{AcCN}$ within $5.4 \mathrm{~min}$; flow rate, $2.64 \mathrm{ml} / \mathrm{min}$; UV detection at 200-300 $\mathrm{nm}$.

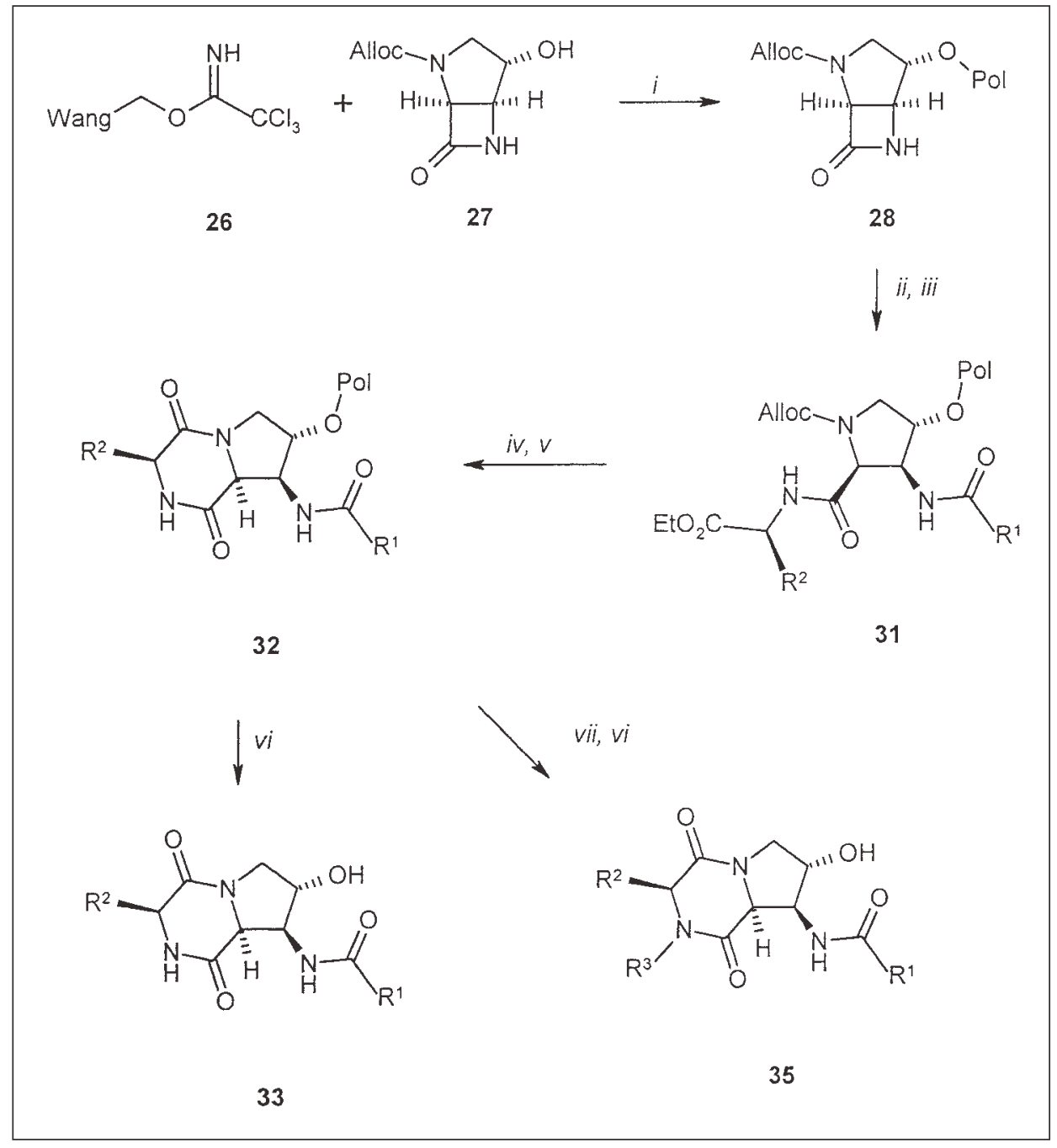

Scheme 3. Reagents and conditions: i) $\mathrm{BF}_{3}{ }^{*} \mathrm{Et}_{2} \mathrm{O}$, $\mathrm{THF} / \mathrm{CH}_{2} \mathrm{Cl}_{2}, \mathrm{rt}-65^{\circ} \mathrm{C}$; ii) $\mathrm{R}^{1}-\mathrm{COCl}$ (29), DIPEA DMAP, DMF, rt; iii) AAs (30), DIPEA, DMF, rt$50{ }^{\circ} \mathrm{C}$; iv) $\mathrm{Pd}\left(\mathrm{PPh}_{3}\right)_{4}, \mathrm{Me}_{2} \mathrm{NH}^{*} \mathrm{BH}_{3}, \mathrm{DMF}, \mathrm{rt}$; v) TMG, DMF, rt-60 ${ }^{\circ} \mathrm{C}$; vi) TFA, $\mathrm{CH}_{2} \mathrm{Cl}_{2}$, rt; vii) $\mathrm{R}^{3}-\mathrm{X}(34)$, phosphazene, DMF, rt-65 ${ }^{\circ} \mathrm{C}$ 


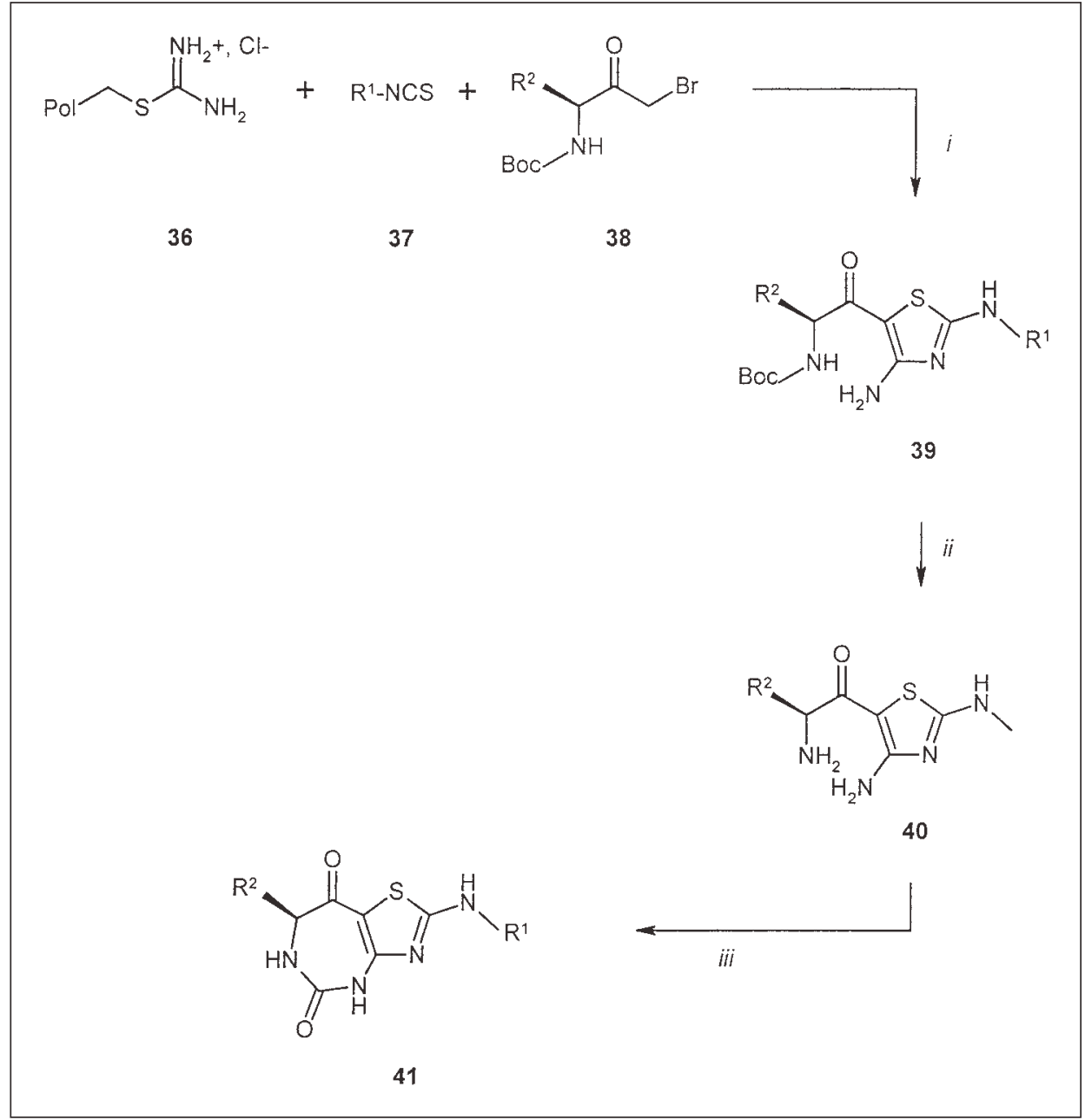

Scheme 4. Reagents and conditions: i) a: 37, DIPEA, DEAMP-resin, DMF, rt; b: 38, DMF, rt; ii) Amberlyst $\mathrm{A}-15, \mathrm{CH}_{2} \mathrm{Cl}_{2}$, rt; iii) $\mathrm{CDI}, \mathrm{Et}_{3} \mathrm{~N}, \mathrm{DMF}$

Table 8. Preparation of 2,4-diaminothiazoles 39 and fused heterocycles 41.

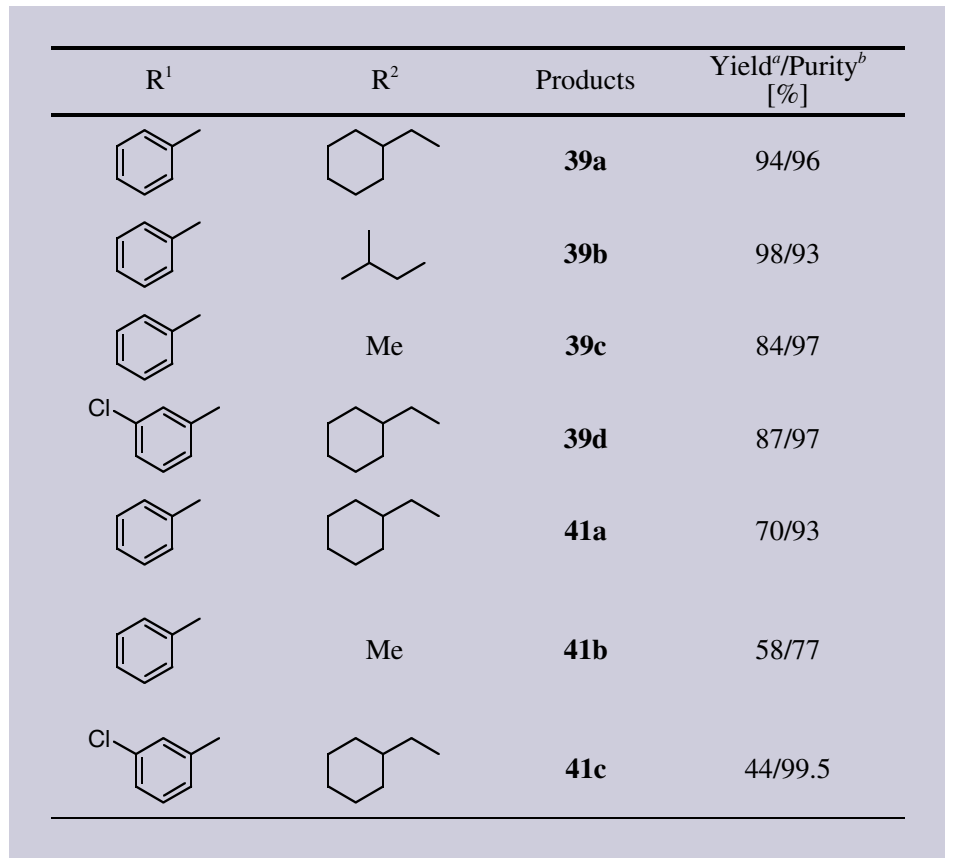

ayields in $\%$ are based on weight of crude material and are relative to the initial loading. ${ }^{b} \mathrm{HPLC}$ Purity of the purified material (confirmed by $\left.{ }^{1} \mathrm{H}-\mathrm{NMR}\right)$, measured on YMC-Pack Pro $\mathrm{C} 18$ column $(75 \times 4.6 \mathrm{~mm})$ with a gradient $12 \% \mathrm{AcCN} / \mathrm{H}_{2} \mathrm{O} \rightarrow 95 \% \mathrm{AcCN}$ within $5.4 \mathrm{~min}$; flow rate, $2.64 \mathrm{ml} / \mathrm{min}$; UV detection at $200-300 \mathrm{~nm}$.

\section{Acknowledgements}

The authors would like to thank L. Bury and A. Gruschwitz for the purification of the libraries, Roche colleagues for analytical results, as well as Prof. M. Page, Dr. G. Schmid, and Dr. M. Roger-Evans for helpful discussions and proof-reading.

Received: March 25, 2003

[1] J.K. Scott, G.P. Smith, Science 1990, 249, 386.

[2] S.E. Cwirla, E.A. Peters, R.W. Barrett, W.J. Dower, Proc. Natl. Acad. Sci. U.S.A 1990, 87,6378

[3] J.J. Devlin, L.C. Panganiban, P.E. Devlin, Science 1990, 249, 404

[4] Z. Xin, Z. Pei, T. von Geldem, M. Jirousek, Tetrahedron Lett. 2000, 41, 1147.

[5] A. Chucholowski, T. Masquelin, D. Obrecht, J. Stadlwieser, J.M. Villalgordo, Chimia 1996, 50, 525.

[6] T. Masquelin, D. Sprenger, R. Baer, F. Gerber, Y. Mercadal, Helv. Chim. Acto 1998, 81, 646.

[7] D. Obrecht, C. Abrecht, F. Grieder, J.-M. Villalgordo, Helv. Chim. Acta 1997, $80,65$.

[8] P. Griffiths, 'Chemical Analysis', John Wiley \& Sons, New York, 1974, Vol. 43.

[9] F.A. Davis, O.D. Stringer, J. Org. Chem. 1982, 47, 1774

[10] H.-S. Ahn, A. Bercovici, G. Boykow, A. Bronnenkant, S. Chackalamannil, J. Chow, R. Cleven, J. Cook, M. Czarniecki, C. Domalski, A. Fawzi, M. Green, A. Gundes, G. Ho, M. Laudicina, N. Lindo, K. Ma, M. Manna, B. McKittrick, B. Mirzai, T. Nechuta, B. Neustadt, C. Puchalski, K. Pula, L. Silverman, E. Smith, A. Stamford, R.P. Tedesco, H. Tsai, D. Tulshian, H. Vaccaro, R.W. Watkins, X. Weng, J.T. Witkowski, Y. Xia, H. Zhang, J. Med. Chem. 1997, 40, 2196.

[11] J.C. Caille, S. Didierlaurent, D. Lefrancois, M.H. Lelievre, C. Sury, J. Aszodi, Synthesis 1995, 6, 635.

[12] H.R. Snyder, L. Katz, J. Am. Chem. Soc 1947, 69, 3140

[13] D.L. Flynn, J.Z. Crich, R.V. Devraj, S.L. Hockerman, J.J. Parlow, M.S. South, S. Woodard, J. Am. Chem. Soc. 1997, 119, 4874.

[14] A. Bianco, J. Furrer, D. Limal, G Guichard, K. Elbayed, J. Raya, M. Piotto, J.-P. Briand, J. Comb. Chem. 2000, 2, 681.

[15] S. Hanessian, F. Xie, Tetrahedron. Lett. 1998, 39, 736; S. Hanessian, F. Xie, Tetrahedron. Lett. 1998, 39, 737.

[16] M. Steger, C. Hubschwerlen, G. Schmid, Bioorg. Med. Chem. Lett. 2001, 11, 2537.

[17] P. Gomez-Martinez, M. Dessolin, F. Guibé, F. Albericio, J. Chem. Soc. Perkin Trans. 1999, 2871. 